\title{
RÖMISCHE MÜNZEN UND DER FRÜHRÖMISCHE GELDUMLAUF BEI DEN ERAVISKERN
}

\author{
MELINDA TORBÁGYI
}

Ungarisches Nationalmuseum

Múzeum krt. 14-16, H-1088 Budapest, Ungarn

torbagyi.melinda@hnm.hu

\begin{abstract}
Coins unearthed in the Eraviscus settlement of Lágymányos provide new data to the beginning of Roman coin circulation in Pannonia. Eravisci are the only Celtic tribe that released coins following the Roman standard at the dawning of the Roman conquest of the area. Exact dating and purpose of this coinage are debated up until now since Celtic denarii do not appear on Celtic settlements, so it seems they played no roles in local coin usage. Meanwhile, Roman bronze coins released in the name of Augustus occur in indigenous settlements. The presence of these Roman bronze coins in the indigenous population in the times of the Iulio-Claudian dynasty prove the existence of relations between the military appearing in the area at that time and the local population.
\end{abstract}

Keywords: Eraviscus, Aquincum, Roman coin circulation, Iulio-Claudian dynasty, Roman conquest of Pannonia

Im Gebiet südlich vom Gellértberg kamen neue frühkaiserzeitliche Siedlungsdetails der einheimischen Bevölkerung an den von József Beszédes zwischen 2007 und 2009 durchgeführten Ausgrabungen ans Tageslicht. ${ }^{1}$ An den Freilegungen kamen auch elf römische Bronzemünzen, die mit Ausnahme von drei Münzen aus der Zeit der Iulius-Claudius-Dynastie stammen, vor.

\author{
1. As des Augustus \\ Rom, 6 v. Chr. \\ RIC 1. 437. (Licinius Nerva Silianus) \\ Inv.-Nr.: 2009.3.11104. \\ SE-176, 721. 2009. 04. 22. - aus einem römischen Brunnen
}

2. As des Augustus

Rom, 16-6 v. Chr.

Avers: Kopf nach rechts

Revers: III VIR AAAFF[

Inv.-Nr.: 2007.19.23310.

Obj. 9 - aus der Auffüllung der Wohngrube (103)

3. As des Augustus

Rom, 16-6 v. Chr.

Avers: Kopf nach rechts

${ }^{1}$ BesZÉDes 2010. 
Revers: III VIR AAAFF[

Inv.-Nr.: 2007.19.23262.

Obj. 737 - neben dem Ofen (3439)

4. As des Augustus

Rom, 11-12

RIC 1. 471.

Inv.-Nr.: 2007.19.7.

Obj. 796, 2007. 10. 31. (3769) - aus der Wohngrube

5. As des Augustus

Rom, 11-12

RIC 1. 471.

Inv.-Nr.: 2007.19.6.

Obj. 796, 2007. 10. 27. (3569)-aus der Wohngrube

6. As des Caligula

Rom, 37-41

Inv.-Nr.: 2007.19.23311.

Obj. 796 - Wohngrube (3570)

7. Römischer As

1.-2. Jahrhundert

Sehr abgenutzt.

Inv.-Nr.: 2007.19.23282.

In der Nähe des Objektes 899 (3670)

8. Sesterz des Claudius

Zeitgenössische „Fälschung“ (Gallien oder Hispanien)

1. Jahrhundert

Inv.-Nr.: 2009.3.11099.

SE-058 (053) 2009. 03. 25. - nahe einem Pfostenloch

9. As des Hadrianus

Rom, 119-138

RIC 2. 679.

Inv.-Nr.: 2009.3.11102.

SE-152, 2009. 04. 20. (645) - unter den Steinen der römischen Straße

10. Constantius II. AE2

348-354

Revers: Fel temp reparatio - Virtus-Typ

Inv.-Nr.: 2009.3.11096.

SE-017, 2009. 03. - in der Auffïllung des Grabens

11. Constantius II. AE3

Siscia, 350-361

Revers: Fel temp reparatio - Virtus-Typ

Inv.-Nr.: 2007.19.23253.

Obj. 719 - auf der römischen Straße 


\section{DIE ÖRTLICHE ERAVISKISCHE MÜNZPRÄGUNG}

Vor der ausführlichen Behandlung der römischen Bronzemünzen sollen wir die örtliche Münzprägung und -benutzung dieses Gebietes, d. h. das wirtschaftliche Milieu, in dem diese Münzen ankamen, überblicken.

Es ist bekannt, dass der Stamm der Eravisker schon am Beginn der römischen Expansion - vielleicht früher - gute Beziehungen mit den Vertretern der in Transdanubien erscheinenden neuen Macht hatte. Die offensichtlichsten Äußerungen dieser Tatsache sind die auf römisches Vorbild, nach den Münzbildern und Münzfuss der römischen republikanischen Denare geprägten Silbermünzen, auf deren einzelnen Typen auch noch der Name des Stammes in Form RAVIS bzw. IRAVISCI vermerkt wurde. ${ }^{2}$ Die genaue Prägungszeit der eraviskischer Denare wurde bis auf den heutigen Tag nicht geklärt. So viel ist aber klar, dass ihre Emission nach der Besetzung von Siscia im Jahre 35 v. Chr. am wahrscheinlichsten ist. Da begann die römische Expansion in Richtung Norden, nach dem Karpatenbecken. Im Laufe dieses Prozesses wurde die Provinz Pannonien allmählich besetzt, okkupiert und zuletzt organisiert. Das geschah am spätesten am Anfang der Regierung des Claudius, obwohl uns immer mehrere Angaben zur Verfügung stehen, wonach dieser Vorgang vielleicht schon während der Regierung des Tiberius begann. ${ }^{3}$ Die etwa 70-80 Jahre, gut zwei Generationen, während sich der Prozess vollzog, blieben aber recht im Dunkeln, da es keine schweren Kämpfe nördlich von der Drau gab und es scheint, dass die Römer von den dort lebenden Volksgruppen, unter ihnen auch von den Eraviskern, friedlich empfangen wurden. Wenige eindeutig aus diesem Zeitalter stammende Denkmäler sind bekannt. Die sich auf dieses Gebiet beziehenden schriftlichen Quellen sind sporadisch und auf vielerlei Art interpretierbar. ${ }^{4}$

In Verbindung mit der eraviskischen Münzprägung und vielleicht auch mit dem späteren Münzverkehr dieses Areals ist die Frage, warum diese Münzen geprägt wurden, am wichtigsten. Das Gros des bekannten Fundmaterials stammt aus Schatzfunden, während praktisch keine einzige Münze in Siedlungsmaterialien und aus archäologischen Freilegungen bekannt ist. Solche Münzen kamen weder im Oppidum auf dem Gellértberg noch in der Tabáner Siedlung zum Vorschein. Auch an der neulich intensiv durchgeführten großflächigen Freilegung der Siedlung von Budaörs wurde kein einziger eraviskischer Denar gefunden. Die eraviskischen Münzen fehlen auch in der ebenfalls gut erforschten Siedlung von Páty, ${ }^{5}$ deren Anfänge - wie die des Vicus von Budaörs - in die späte Eisenzeit zu datieren sind. Nur in der Umgebung der Siedlung wurde eine einzige quinar-Nachahmung als Streufund gefunden. ${ }^{6}$ Aber auch noch die den Eraviskern zuzuschreibenden quinar-Funde - obwohl sie sehr selten auftauchen - kommen eher in Schatzfunden vor. Solche sind der eraviskische Münzfund von Devin (Dévény) und der in der Umgebung von Győr. ${ }^{7}$ Der am spätesten datierbare Münzschatz (Bia) schließt mit der Münze des Caligula. ${ }^{8}$ Diesmal möchte ich bemerken, dass fünf römische republikanische Denare und ein Legionsdenar des M. Antonius in den Trümmern eines ins 1. Jahrhundert n. Chr. datierten niedergebrannten Hauses und eines Ofens in der einheimischen Siedlung von Páty ans Tageslicht kamen. ${ }^{9}$

Der Anfang und die Veranlassung der Münzprägung wurden von einigen Forschern mit dem dakischen, gegen die Boii geführten Feldzug in Verbindung gebracht, ${ }^{10}$ weil es römische Münzen, die auch nachgeahmt wurden, schon in der ersten Hälfe des 1. Jahrhunderts v. Chr., sogar vielleicht auch früher, im Gebiet Daziens in großer Zahl gab. Die Nachahmungen können von den ursprünglichen Stücken häufig nur schwer unterschieden werden. ${ }^{11}$ Im eraviskischen Fundmaterial gibt es zweifellos auf dakische Anwesenheit hinweisende Gegenstände (sog. dakische Keramik), aber weder eine massive Anwesenheit noch eine Verbindung mit dem dakischen Staat, die den Zufluss von römischen republikanischen Denaren in großer Menge begründen könnten, sind bekannt. ${ }^{12}$ Im Gebiet der heutigen Slowakei, das viel intensiver unter dakischem Einfluss stand, sogar von den Daken besetzt und bewohnt war, ist gar keine Übung zur nach dakischem Muster geübten Münzprägung bekannt. Ein Teil der sporadisch vorkommenden Nachahmungen der republikanische Denare kann zweifellos an die dakische Expansion geknüpft werden, aber keine systematische Münzprägung nach römischem Vorbild wurde im erwähnten Gebiet ausgeübt. ${ }^{13}$ Ferner wurden zahlreiche, von slowakischen Fundorten bekannte republikanische Denar-Nachahmungen schon

\footnotetext{
2 TORBÁGYi 1984.

${ }^{3}$ GABLER 2006

${ }^{4}$ Dieser Fragenkreis wurde neulich zusammenfassend überblickt bei KovÁcs 2014, 23-57.

${ }^{5}$ TORBÁGYI 2007.

${ }^{6}$ TORBÁGYi 2007, 277, 286.

${ }^{7}$ Torbágyi 2005; Plachá-Fiala 1998; HaUPT-NiCK 1997.
}

${ }^{8}$ J. ECKHEL: Doctrina Numorum Veterum. Vindobonae 1792-1798. Das wird auch von späteren Verfassern wie Ö. Gohl zitiert (GoHL 1902, 31).

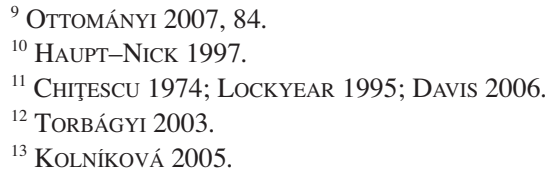


zweifelsohne nach eraviskischem Vorbild geprägt. ${ }^{14}$ Auch bei den am Rand des Nördlichen Mittelgebirges vorgekommenen römischen republikanischen Münzschätzen ist das Ankommen von Dazien her eine der Möglichkeiten. ${ }^{15}$ Eine Münzprägung nach römischem Muster ist viel mehr in einem römischen wirtschaftlich-politischen Milieu vorstellbar. Einige vor der Besetzung von Siscia im Jahre 35 v. Chr. angefertigte gegenständliche Denkmäler weisen schon auf die Beziehungen vor der Besitznahme hin. ${ }^{16}$

Die römischen politischen Versuche, die Verbindungen mit den zu erobernden Gebieten aufzunehmen und die örtliche Elite mit Luxusartikeln und Geld zu gewinnen, richteten sich auf die Anschaffung der Unterstützung der einheimischn führenden Schicht. Die Exemplare des in Mucsi (Kom. Tolna) ${ }^{17}$ vorgekommenen bronzenen Münzschatzes eigenartiger Zusammensetzung sind aber in diesem Kontext nicht auslegbar. In diesem Ensemble überraschender Zusammensetzung befinden sich die Reliquien mindestens zweier Epochen. Die beiden ältesten Münzen - die vom König Agathokles von Syrakus um 310 v. Chr. geprägte hemilitra und die zwischen 217 und 215 v. Chr. datierbare uncia mit prora - könnten noch durch die mediterranen Verbindungen der keltischen Epoche nach Transdanubien gelangt haben. Aber vier Bronzemünzen römischer und eine Münze syrischer Prägung des Augustus, ferner der as des Tiberius können schon an die Zeit der römischen Eroberung geknüpft werden. Nach der Behauptung des Monumentum Ancyranum 30 wurden die Grenzen des Reichs von Augustus an der Donau gezogen, auch wenn das zwangsläufig nicht die tatsächliche Besetzung des Gebietes bedeutete. Mit den friedlichen Eraviskern als Verbündeten ist eine Verbindung, in deren Rahmen die tammesführsten Geld erhielten und nach diesem Muster auch sie selbst mit einer eigenen Geldemission begannen, nicht auszuschließen. ${ }^{18}$

In den anfänglichen Jahrzehnten der römischen Herrschaft existierten noch zahlreiche heimische Münzprägungen - auch aus Edelmetall - im Gebiet des von Iulius Caesar eroberten Gallien und diese Münzen konnten bei der Besoldung der im römischen Dienst stehenden heimischen Hilfstruppen benutzt werden. ${ }^{19}$ Aufgrund der Verbreitung und des Vorkommens der Münzen dürften eher die Politik und die Wirtschaft auch die eraviskische Münzprägung veranlasst haben. In der selbstständigen Münzprägung kommt einerseits das stämmische Selbstbewusstsein zum Ausdruck, zugleich ist es nicht auszuschließen, dass das vorrangige Ziel die Finanzierung der mit den Römern verbündeten eraviskischen Truppen in dem gegen Marobodus geplanten Krieg war. So könnten die in Devin (Dévény) und in der Umgebung von Győr gefundenen eraviskischen Münzfunde, ferner zahlreiche, im Gebiet der heutigen Slowakei vorgekommene Streufunde, gut erklärt werden. ${ }^{20}$ Eine eraviskische Münze (Torbágyi 1984, $\mathrm{C} / 12 \mathrm{i}$ ) gelangte ganz gewiss durch die Vermittlung von römischen Soldaten nach der Rheinland (Barenaue-Lutterkrug), mit römischen Denaren gemischt. ${ }^{21}$ Natürlich kann der Handel als eine Möglichkeit ebenfalls nicht ausgeschlossen werden. Die in der Po-Ebene gefundenen eraviskischen Denare ${ }^{22}$ können vielleicht beweisen, dass die örtliche Stammeselite schon als Abnehmer von Luxusartikeln auftrat.

Das Existieren der eraviskischen Münzprägung bedeutet aber nicht, dass man vor der Römerzeit, sogar an deren Anfang, in ihrem Stammesgebiet mit einem örtlichen Münzverkehr rechnen kann. Was versteht man eigentlich unter dem Begriff Münzverkehr? Die Kenntnis der Münzen, aber auch noch das Existieren einer eigenen Münzprägung bedeuten nicht unbedingt, dass man mit Münzverkehr in einer Gemeinschaft rechnen soll. Es ist kein Zufall, dass die Begriffe „Münzverkehr“ und „Münznutzung“ (coin use) heutzutage von den Forschern in der Fachliteratur unterschieden werden. Die verschiedenen Völker des späteisenzeitlichen Europa kannten, sogar produzierten auch selbst Münzen, die als Prestigegegenstände, repräsentative Geschenke, Opfer, Zahlungsmittel anlässlich von Feldzügen dienten und natürlich auch im Fernhandel bei den Auszahlungen ansehnlicher Summe eine Rolle spielten ${ }^{23}$ In der Wirklichkeit induzierte das Erscheinen der Oppida, der stadtartig angelegten Siedlungen den

${ }^{14}$ KolníKOVÁ 2005, 80, Abb. 2.

${ }^{15}$ TORBÁGYI-VIDA 2013.

${ }^{16}$ GABLER 2005.

${ }^{17}$ ALFÖLDI 1946. Es handelt sich nicht um eine geschlossene Fundeinheit. Auf einen Schatzfund weist nur die Tatsache hin, dass die Münzen nach der Mitteilung von Mérey einander nahe ans Tageslicht kamen. Am nördlichen Rand der Großen Ungarischen Tiefebene ist ein Schatzfund, dessen Münzen ganz bestimmt aus zwei verschiedenen Epochen stammen, in Abasár (Kom. Heves) bekannt. Es ist vorstellbar, dass die 9 keltischen Silbermünzen, die 39 republik- und 4 kaiserzeitlichen Denare, von denen eine Traianus-Münze am spätesten $\mathrm{zu}$ datieren ist, das durch Generationen gesammelte Vermögen einer nicht zu reichen Familie gewesen war (TORBÁGYI-VIDA 2013).
${ }^{18}$ ToRbÁGYI 1997-1998, 14; KovÁCs 2005, 224; KovÁCS 2014, 55.

${ }^{19}$ WightMAN 1974, 481; WighTMAN 1977; WOLTERS 1988 - In erster Linie auf historischem Grund wird diese Theorie ausführlich kritisiert; siehe WIGG 2002, 226; HEesch 2005, 236-237; LOSCHEIDER 2005, 119-124. Die lange Debatte in diesem Thema ist noch nicht abgeschlossen, aber das kann im Rahmen dieser Studie nicht ausführlich erörtert werden. 2006, 72-76

${ }^{20}$ Plachá-Fiala 1998; Haupt-Nick 1997; KolníKová

\footnotetext{
${ }^{21}$ BERGER 1992, 244, Kat. 158.

${ }^{22}$ Revue Numismatique 1860, 203.

${ }^{23}$ NICK 2006.
} 
Beginn des tatsächlichen Münzverkehrs, wobei die nicht mehr autarken Bewohner im Alltagsleben, auch bei kleinen Einkäufen, Geldmünzen brauchten. ${ }^{24}$ Deshalb erschienen die verschiedenen Kleingelder, Silbermünzen niedrigen, sogar immer niedrigeren Wertes und die nicht aus Edelmetall hergestellten Münzen, also die gegossenen und geprägten Kupfer- bzw. Bronzemünzen. Das Existieren eines differenzierten Währungssystems kann auf jeden Fall als das sichere Zeichen einer entwickelteren Geldnutzung, eines sich entfaltenden Münzverkehrs bewertet werden. ${ }^{25}$ Die auf eraviskischem Territorium vorgekommenen wenigen Quinare können aber nur als der Beginn eines in diese Richtung zeigenden Prozesses aufgefasst werden. Dieser Prozess schloss sich mit dem Erscheinen der Römer - genauer: er wurde mit dem Erscheinen der Römer voll entfaltet. ${ }^{26}$

\section{FRÜHKAISERZEITLICHE BRONZEMÜNZEN IN DEN ERAVISKISCHEN SIEDLUNGEN}

In der Siedlung von Lágymányos sind die in Rom geprägten sog. triumviri monetales-Asse des Augustus die ältesten Bronzemünzen, die zwischen 18 und $6 \mathrm{v}$. Chr. produziert wurden, ferner ein ebenfalls in Rom 11-12 n. Chr. emittierter As. Im Vicus von Budaörs sind fünf Asse des Augustus bekannt, in der Siedlung von Páty vier Stücke und ein zwischen 10 und 12 geprägtes Exemplar des Tiberius. Obwohl die Prägungszeit der römischen Münzen recht exakt bestimmbar ist und auch noch die abgenutzten Exemplare in relativ engen Grenzen datierbar sind, konnten sie viel länger im Verkehr sein. Früher dachte man, dass die Benutzung und der Verkehr der Bronzemünzen - da die Bronze kein wertbeständiges, für eine lang anhaltende Thesaurierung geeignetes Edelmetall ist nur auf eine kurze Zeit zu beschränken sind. Also kann ein während der Regierung des Augustus emittierter As vermutlich in die augusteische Zeit datiert werden. Heute beweisen aber schon zahlreiche Angaben, dass das gar nicht so sicher ist, besonders nicht in den ersten Jahrzehnten der Kaiserzeit. Eben in diesem Zeitabschnitt ist die lange, wegen des für diese Periode kennzeichnenden Mangels an Kleingeld beobachtbare Verkehrszeit der Bronzemünzen charakteristisch. Bei den gut datierbaren Militärlagern in der Rheinland kann man nachweisen, dass die später, zwischen 7 und 5 v. Chr., emittierten Serien der augusteischen triumviri monetales-Asse ${ }^{27}$ in den nach dem Tod des Augustus angelegten Lagern (z. B. Vindonissa) ${ }^{28}$ viel häufiger auftreten. Nördlich von den Alpen kann man aber im Allgemeinen beobachten, dass sie den Höhepunkt ihres Verkehrs - gewöhnlich kontramarkiert - während der Regierungszeit des Tiberius erreichten. ${ }^{29}$ Durch die Münzen in sich selbst können also ein Objekt, ein Ort nicht genau datiert werden. Sie können nur als terminus post quem in Betracht gezogen werden. Als sicherer zeitlicher Anhaltspunkt können die Münzen nur in dem Fall dienen, wenn andere mit ihnen vorkommende, gut datierbare Gegenstände - zum Beispiel Sigillaten - das unterstützen.

Augusteische Bronzemünzen kamen auch im Gebiet des zum ala-Lager der Wasserstadt/Víziváros (das beweisbar das älteste militärische Objekt von Aquincum ist) gehörenden Vicus ans Tageslicht. ${ }^{30}$ Zwei augusteische Asse und ein nicht an Ort hergestelltes keltisches Kleinsilber kamen unter den Trümmern eines ins 1. Jahrhundert datierten, Reparaturwerkstatt genannten Gebäudes mit den Bruchstücken von frühen Bronzegegenständen zum Vorschein. ${ }^{31}$ An den 2000 und 2001 durchgeführten Ausgrabungen des Vicus des ala-Lagers kamen noch drei Asse und ein quadrans vor. ${ }^{32}$ Im Vicus des ala-Lagers kamen des Weiteren zwei Tiberius- und ebenfalls zwei Tiberius Divus Augustus-Asse, einer von ihnen noch an den alten Freilegungen des Thermalbades Király (Királyfürdö), ans Tageslicht. ${ }^{33}$ Unter den alten Münzen ist auch ein Agrippa-As zu finden (RIC 58, der heute auch für eine Prägung von Caligula gehalten wird). Trotz der Geldmünzen kann man mit der Anlegung des Lagers aufgrund sowohl des archäologischen Fundmaterials als auch der Truppengeschichte erst von der claudischen Zeit an rechnen. ${ }^{34}$

\footnotetext{
${ }^{24}$ NASH 1976.

${ }^{25}$ TORBÁGYi 2008, 205.

${ }^{26}$ TORBÁGYI 2008.

${ }^{27}$ KRAFT 1955-1956.

${ }^{28}$ KRAAY 1962

${ }^{29}$ HeEsch 2000, 158. Diese Verteilung kann auch im Münzmaterial des am frühesten in der späten tiberianischen Zeit (GABLER 2006) angelegten Lagers von Carnuntum beobachtet werden:
} VONDROVEC 2007, 93.
${ }^{30}$ H. KÉRDỎ 1997; H. KÉRDỎ 2003.

${ }^{31}$ H. KÉRDÕ 2011, 168.

${ }^{32}$ Quadrans Rom 9 v. Chr. RIC 422; as Rom 7 v. Chr. RIC 428; as Rom 7 v. Chr. vgl. RIC 431, 435; abgenutzter as Lugdunum 10-14 v. Chr. Ausgrabungen von K. H. Kérdő und P. Kovács, TölgyfaStr. 3. - Henger-Str. 2.

${ }^{33}$ Münzfunde: NK 5 (1906) 141

${ }^{34}$ GABLER 1971; GABLER 1997; H. KÉRDŐ 2011. 
Im Vicus des Lagers kenne ich neun Münzen von Claudius, also einen Denar, einen Sesterz, sechs Asse und eine zeitgenössische Fälschung. ${ }^{35}$

Von den augusteischen Münzen von Lágymányos kamen drei Exemplare mit Keramikbruchstücken (Kat. 2, 4-5) ans Tageslicht: Zwei Stücke (Kat. 4-5) wurden in der Auffüllung von durch Sigillaten in die claudischflavische Zeit datierten Wohngruben gefunden und ein Exemplar kam in der Auffüllung einer Wohngrube, die ebenfalls claudisch-flavische Sigillaten, ferner streifenbemalte Keramik von Brigetio und marmorierte Keramik enthielt (Kat. 2), vor. Der Kontext der Asse von Budaörs und Páty ist leider ungewiss oder es gibt gar keinen Kontext, demnach verfügen diese Münzen nur über einen winzigen Datierungswert. In der Siedlung von Lágymányos kamen ein abgenutzter As des Caligula in Vergesellschaftung von ebenfall in die claudisch-flavische Zeit datierten Sigillaten in einer Wohngrube (Kat. 6), ferner die zeitgenössische Fälschung eines Sesterzes des Claudius in der Nähe eines Pfostenlochs (Kat. 8) zum Vorschein. Auch in der Eraviskersiedlung von Tabán ist eine Münze des Claudius bekannt. Diese Münze könnte zugleich auch das Ende der Siedlung mit dem Bruchstück einer an Ort hergestellten Schüssel der Form Drag. 37 bezeichnen. ${ }^{36}$ In der Siedlung von Páty fand man sechs Asse des Tiberius (unter ihnen zwei von Divus Augustus und zwei von Drusus), einen As des Caligula, einen Sesterz des Claudius, ferner dessen zeitgenössische Nachahmung und ein Exemplar des Germanicus, leider ohne Kontext als Streufund auf der Oberfläche. ${ }^{37}$ Im Vicus von Budaörs sind je ein As des Tiberius und Divus Augustus und zwei Agrippa-Asse des Caligula bekannt. Zum Vorschein kamen noch ein Antonia-Dupondius und ein Nero Drusus-Sesterz des Claudius, ferner drei sehr abgenutzte kontramarkierte, in die Zeit der Iulius-Claudius-Dynastie datierbare Asse. ${ }^{38}$

Nach der Münze des Claudius gibt es einen langen Hiatus in der Siedlung von Lágymányos, dann ist der nächste Fund ein unter den Steinen der römischen Straße vorgekommener As des Hadrianus (Kat. 9). Das vollkommene Fehlen der Münzen der flavischen Zeit scheint überraschend zu sein. Betrachtet man aber die Münzen des Vicus des Ala-Lagers der Wasserstadt/Víziváros, dann sehen wir, dass es auch dort wenige Exemplare im Vergleich zu den früheren Münzen gibt. Diese sind also zwei Titus-Münzen des Vespasianus (ein Denar und ein Dupondius) und zwei abgenutzte Asse des Domitianus. Aus dieser Zeitspanne stammt ferner ein kleiner Aureusschatz, der eine Tiberiusund eine Claudius-Münze, dann fünf, nach der Reform emittierte Aurei des Nero enthält und die letzten zwei Münzen sind die Prägungen des Vespasianus aus den Jahren 70 und $71 .{ }^{39} \mathrm{Im}$ Material des Vicus von Budaörs ist eine einzige Domitianus-Münze, eine provinziale Prägung, zu finden. ${ }^{40}$ Auch in der Siedlung von Páty gelten die flavischen Münzen (ein früher Denar des Vespasianus, ein Denar des Domitianus und ein Dupondius) als eine große Seltenheit. ${ }^{41}$

\section{BEWERTUNG}

Es ist eine Frage, wann und wie die vorgekommenen römischen Bronzemünzen in die Eraviskersiedlungen gelangten. Die Bronzemünzen können nämlich weder im Fernhandel noch als Mittel der politischen Beeinflussung betrachtet werden. Ihre Benutzung kann an die Alltage geknüpft werden und im Hintergrund ist durchaus schon ein das Geld intensiv benutzendes Milieu anzunehmen. Das konnte am Anfang der Römerzeit auf dem nordöstlichen Teil Transdanubiens nichts anderes als das römische Militär sein. Das römische Militär wurde schon ab der Mitte des 2. Jahrhunderts v. Chr. in Silber besoldet und aller Wahrscheinlichkeit nach ist die zu dieser Zeit beginnende kräftige Denarprägung dieser Tatsache zu danken. ${ }^{42}$ Die Soldaten brauchten aber auch Kleingeld, da ein Denar 16 Asse galt und man schon in einer Kneipe von Pompei etwa einen halben Liter Wein für einen As trinken konnte. ${ }^{43}$ Dass die Ausstattung mit Kleingeld ein großes Problem war, beweisen die in der Rheinland durchgeführten inten-

\footnotetext{
${ }^{35}$ WIGG 1996, 424-431. In der claudischen Zeit, vielleicht auch schon früher, wollte man den Mangel an Kleingeld durch die Prägung von Nachahmungen verschiedenen Typs in Gallien und vielleicht auch in Hispanien ersetzen. Diese Gepräge sind regelmäßig leichter und kleiner als die offiziellen Exemplare. Durch den Münzverkehr wurden sie in Europa überall verbreitet, so ist ein Stück auch in der heimischen Siedlung von Páty zu finden (TORBÁGYI 2007, 281 bzw. Kat. 68). Obwohl ich die von F. Redő bestimmten Münzen der Mansio von Bátaszék-Lajvér nicht sah, sind unter ihnen meiner Meinung nach zwei oder drei Exemplare zu finden, die aufgrund ihrer Größe ebenfalls zeitgenössische Nachahmungen sein können.
}

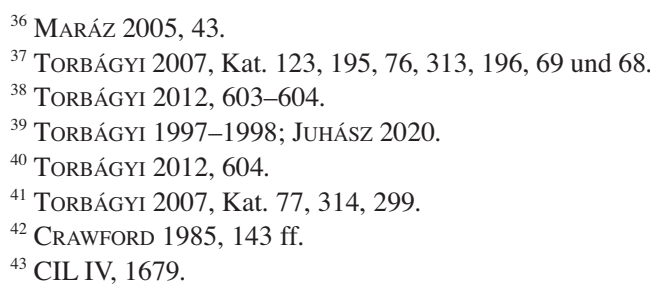

${ }^{36}$ MarÁz 2005, 43.

38 TorbáGYi 2012, 603-604.

39 TORBÁGYi 1997-1998; JuHÁSZ 2020.

40 TORBÁGYI 2012, 604

${ }^{42}$ CRAWFORD 1985, $143 \mathrm{ff}$.

${ }^{43}$ CIL IV, 1679 
siven Freilegungen und Forschungen sehr gut. Zahlreiche halbierte und in noch kleinere Teile geschnittene Bronzemünzen weisen auf die sehr frappante Lösung des Problems hin. ${ }^{44}$ Zwar die Bronzeprägung eben infolge der augusteischen Reform in Rom wieder begann, scheint es, dass der Staat die Ansprüche auf Kleingeld nicht befriedigen konnte. Dementsprechend konnten alte, schon seit einem Jahrhundert außer Gebrauch stehende Münzen in Umlauf gesetzt werden. Man kennt aus dem 2. Jahrhundert v. Chr. stammende, in frühkaiserzeitlichen Militärlagern vorgekommene Asse und ihre verschiedenen Varianten kennzeichnend in der Rheinland, obwohl diese Münzen in einer früheren Epoche in keltischem Milieu unbekannt sind und der Höhepunkt ihres Verkehrs in die tiberischclaudische Zeit datierbar ist. ${ }^{45}$

Aus der frühen Periode der römischen Herrschaft in Pannonien verfügt man nur über ein kleines Münzmaterial, das für eingehende Analysen nicht geeignet ist. Gewisse Tendenzen, die sich gewissermaßen an die Folgerungen der Analysen von auf anderen Teilen des Reiches in größeren Siedlungen bzw. Lagern bekannten, aus mehreren hundert oder tausend Exemplaren bestehenden Münzreihen anknüpfen, können aber auch in diesen Fällen beobachtet werden. Die in der Wasserstadt/Víziváros im Ala-Lager des Vicus vorgekommenen Münzfunde sind die Reste des Soldes des Militärs, da es - wie oben behandelt - keinen örtlichen Münzverkehr vor der römischen Anwesenheit gab. Obwohl die Soldaten grundlegend in Silber, gelegentlich vielleicht in Gold (wie darauf auch der oben erwähnte kleine Aureusfund hindeutet), besoldet waren, sollten sie aber für die zu ihrem Alltagsleben, zu kleineren Einkäufen ganz gewiss nötigen Kleingelder sorgen. Das könnte zentral geschehen sein. Der Sold wurde vielleicht teils in Bronze gezahlt oder durch die Vermittlung von römischen Händlern kamen sie zu Wechselgeld. Die Händler brachten solche Waren mit, mit denen die Örtlichen die Soldaten nicht versorgen konnten.

Auch die Rolle der offiziellen Geldwechsler könnte sich erheben - aber es ist vielleicht zu früh, zu dieser Zeit besonders hier, in dem sehr weit entfernt liegenden und neu angegliederten Teil des Reiches daran zu denken. Die in der Rheingegend sehr sorgfältig durchgeführten numismatischen und archäologischen Forschungen beweisen, dass die Bronzemünzen an den meisten Orten mit dem römischen Heer erschienen oder seine Anwesenheit die Ansprüche der Zivilbewohner auf diese Münzen verursachte - und das konnte gelegentlich auch örtliche Münzprägungen induzieren. ${ }^{46}$ In die heimischen Siedlungen konnten die römischen Münzen durch die Vermittlung des örtlichen Militärlagers gelangen. Und wenn die alten Prägungen im Münzmaterial der Lager lange in Gebrauch blieben, ist das für die Zivilsiedlungen eher noch kennzeichnend. In der oberen Rheingegend kamen zwischen 1985 und 199248 Münzen, von ihnen 29 Exemplare in verhältnismäßig gut bestimmbaren Fundzusammenhängen, an den Freilegungen des Vicus Petinesca ans Tageslicht. Demnach stellte es sich heraus, dass die unter den Trümmern gefundenen Münzen regelmäßig um mehrere Jahrzehnte älter sind als die Datierung des vollkommenen Komplexes. Bei den Häusern ist das Bild schon bunt, weil auch die mit den anderen Gegenständen ungefähr gleichzeitigen Münzen da anwesend sind (z. B. eine Caligula-Prägung in einem um 40-50 datierten Fundensemble). Es kommt aber ebenfalls vor, dass eine augusteische Münze in einem claudiuszeitlichen Material aufzufinden ist. ${ }^{47}$

Das Material der rheinländischen Lager beweist die lange Verkehrszeit der augusteischen Münzen zweifellos, aber für ihre Umlaufsetzung ist es eben kennzeichnend, dass die Bronzemünzen in der Regel einander schnell ablösten.$^{48}$ Das kann mithilfe des Fundmaterials der kurzlebigen und gut datierbaren Lager gut bewiesen werden. Außer dem so genannten Oberaden- und Haltern-Horizont kann neulich auch ein noch älterer Horizont, nämlich der Nijmegen-Horizont, für den verschiedene Emissionen charakteristisch sind, festgestellt werden. ${ }^{49}$

Später, zum Beispiel im Material von Vindonissa, zeigen aber die meisten triumviri monetales-Asse des Augustus schon mit Kontramarken. ${ }^{50}$ Heftig debattiert man, in welchen Geldern das römische Heer besoldet wurde. ${ }^{51}$ Aus den geschichtlichen Quellen wissen wir nur, wie viel Sold, regelmäßiges stipendium ${ }^{52}$ bzw. andere Geldbei-

\footnotetext{
${ }^{44}$ Chantraine 1982. Einige Exemplare sind auch noch in Carnuntum bekannt: VONDROVEC 2007, 97-99.

${ }^{45}$ KraAy 1962; Peter 2001, 40-44. Auch in Carnuntum gibt es einige Exemplare: VONDROVEC 2007, 90-91.

${ }^{46}$ WIGG 2002; RoyMANS-AARTS 2009. - Interessante Gedanken darüber, dass die Münzprägung nicht nur durch wirtschaftliche Gründe induziert werden konnte, sondern dabei auch gesellschaftliche kulturelle und ideologische Faktoren berücksichtigt werden sollen.

${ }^{47}$ FREY-KUPPER 2002.

${ }^{48}$ WIGG 1997, 282-284.

${ }^{49}$ KeMMERs 2006, 246-247
}

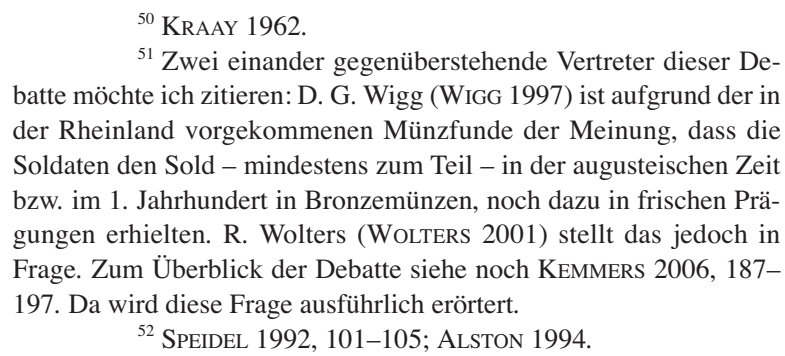

${ }^{51}$ Zwei einander gegenüberstehende Vertreter dieser Debatte möchte ich zitieren: D. G. Wigg (WIGG 1997) ist aufgrund der in der Rheinland vorgekommenen Münzfunde der Meinung, dass die Soldaten den Sold - mindestens zum Teil - in der augusteischen Zeit bzw. im 1. Jahrhundert in Bronzemünzen, noch dazu in frischen Prägungen erhielten. R. Wolters (WOLTERS 2001) stellt das jedoch in Frage. Zum Überblick der Debatte siehe noch KEMMERs 2006, 187197. Da wird diese Frage ausführlich erörtert

${ }^{52}$ SPEIDEL 1992, 101-105; AlSTON 1994. 
träge ${ }^{53}$ die Soldaten bekamen. Die Quellen erwähnen aber nicht, in welchem Metall (Gold, Silber, Bronze), in welchen „Nominalien“ diese Summen gezahlt wurden, ob die vollkommene Summe überhaupt in das Lager gelangte oder die Kosten der Versorgung schon von vornherein zurückbehalten wurden ${ }^{54}$ ferner ob der Sold in alten oder neuen Prägungen gezahlt wurde. Eine weitere Frage ist, woher das Geld zum Militär gelangte: unmittelbar aus Rom oder dem provinziellen Zentrum bzw. unmittelbar zu den einzelnen Truppen oder zum militärischen Befehlshaber des Territoriums. Es ist gar nicht sicher, dass das Versorgungssystem überall gleichermaßen funktionierte.

Die das Ala-Lager der Wasserstadt/Víziváros anlegende Truppe, die ala I Hispanorum kam aus dem dalmatischen Burdum an. ${ }^{55}$ In der Kasse des Lagers oder in der „Tasche“ der Soldaten waren wahrscheinlich auch noch frühere, augusteische Bronzemünzen, die von ihrem älteren Stationsort mitgebracht wurden. Es ist ebenfalls vorstellbar, dass frühere Münzen in der „Kasse“, aus der die Soldaten der ala der Wasserstadt/Víziváros bezahlt wurden, in größerer Menge gewesen sein dürften, da ein Teil der zum Heer einmal schon hingelangten Gelder im Kreis des Militärs ganz gewiss erhalten blieb. Denkt man an die in der tiberischen Zeit kontramarkierten AugustusBronzen, stammten sie offensichtlich aus dem Münzmaterial der Schatzkammer des Staates - vielleicht der militärischen Lager -, da die Gegenstempelung in sich auf die Herkunft aus einem offiziellen Milieu hinweist. Die Emissionen der Iulius-Claudius-Dynastie in Bronze waren nämlich recht unregelmäßig. Nach der an das Ende der 20er Jahre v. Chr. datierten Reform des Augustus begann zwar eine recht massive staatliche Münzprägung in den Münzstatten von Rom, Nemausus und Lugdunum für die Kleingeldversorgung Italiens und der westlichen Territorien, aber nach $4 \mathrm{n}$. Chr. gab es nur sehr wenige Emissionen, abgesehen von den Jahren zwischen $10 \mathrm{und} 14 \mathrm{n}$. Chr., aber im Vergleich zu den früheren Jahren war das viel kleiner. Während der Regierung des Tiberius war die Emission unregelmäßig. In der claudischen Zeit war die Anzahl der neuen Münzen nach einem kräftigen Anfang sehr klein und das Gros der Letzteren war so genannte irreguläre Prägung. ${ }^{56}$ Um eine regelmäßige Bronzeprägung handelt es sich nur nach der Reform des Nero in den Jahren 61 und 62. In der tiberisch-claudischen Zeit ist also das Erscheinen von zahlreichen so genannten irregulären Bronzemünzen, die überwiegend in Gallien, aber vielleicht auch anderswo geprägt wurden, kein Zufall. ${ }^{57}$ Dass die frühen augusteischen Bronzen auf dem nordöstlichen Transdanubien aus dem zivilen Verkehr - durch römische Händler und Handwerker, die hinter dem Heer kamen und dessen Versorgung sicherten - zu den im Lager lebenden Soldaten gelangt hätten, ist in der Tat da und zu dieser Zeit meiner Meinung nach noch nicht wahrscheinlich.

Zur einheimischen Bevölkerung gelangten die römischen Bronzemünzen durch die Verbindungen mit dem Militär. Darauf weist die ähnliche Verteilung der im Vicus des Lagers der Wasserstadt/Víziváros und in den Eraviskersiedlungen anwesenden Münzen. Die Verbindung der örtlichen Bewohner und der Soldeten beweisen dann die Funde, d. h. die in den Zivilsiedlungen auftauchenden Militaria, ab der zweiten Hälfte des 1. Jahrhunderts immer mehr. ${ }^{58}$ Auf die Romanisation dieses Raumes - wie auch auf die Romanisation anderer Territorien - wirkte das römische Heer unbestreitbar fördernd. ${ }^{59}$

\section{LITERATUR}

ALFÖLDI 1946

ALSTON 1994

BERGER 1992

BERGER 1996

BESZÉDES 2010

\begin{abstract}
= A. ALFÖLDI, jun.: A Dunántúl felé terjeszkedő kora római kereskedelem új nyomai (Neue Spuren des sich nach Transdanubien ausdehnenden frührömischen Handels). MMúz 2 (1946) 52-57.

= R. ALSTON: Roman military pay from Caesar to Diocletian. JRS 84 (1994) 113-123.

= F. BERGER: Untersuchungen zu römerzeitlichen Münzfunden in Nordwestdeutschland. Studien zu Fundmünzen der Antike 9. Berlin 1992.

= F. BERGER: Kalkriese I. Die römischen Fundmünzen. RGF 55. Mainz am Rhein 1996.

= J. BEsZÉDEs: Újabb kora császárkori teleprészlet Lágymányoson - Part of a new Imperial period settlement at Lágymányos. AqFüz 16 (2010) 113-118.
\end{abstract}

${ }^{53}$ JAHN 1984, 54-55.

${ }^{54}$ Kemmers 2006, 192. Folgendes Werk wird zitiert: R. O. Fink: Roman Military Records on Papyrus. Philological monographs of the American Philological Association 26. Cleveland 1971.

${ }^{55}$ LỎRINCZ 1990, 75.

\author{
${ }^{56}$ Siehe KeMMERs 2006, 90-92, mit ausführlicher Literatur \\ über die claudischen Bronzen. \\ ${ }^{57}$ KING 1996, 239-246; WIGG 1996, 424-434. \\ ${ }^{58}$ MrÁv 2012. \\ ${ }^{59}$ Mráv 2004; Hanel 1999.
}


BESZÉDES-HORVÁTH 2008

ChanTRAINE 1982

CHIŢESCU 1981

CRAWFORD 1985

DAVIS 2006

FREY-KUPPER 2002

GABLER 1971

GABLER 1997

GABLER 2005

GABLER 2006

GABLER 2012

GoHL 1902

HABLE 1998

HANEL 1999

HAUPT-NICK 1997

HEESCH 2000

HeESCH 2005

HEINRICHS 2000

JAHN 1984

JUHÁSZ 2020

KEMMERS 2006

H. KÉRDŐ 1997

H. KÉRDŐ 2003
= J. BESZÉDES-L. A. HorvÁth: Ôskori és római kori lelőhelyek a Budai Skála bontása során - Prehistoric and Roman period sites uncovered during the demolition of the Budai Skála department store. AqFüz 14 (2008) 141-157.

= H. Chantraine: Die antiken Fundmünzen von Neuss. Gesamtkatalog der Ausgrabungen 1955 1978. Novaesium 8. Limesforschungen 20. Berlin 1982.

= M. Chiţescu: Numismatic Aspects of the History of the Dacian State. The Roman Republican Coinage in Dacia and Geto-Dacian Coins of Roman Type. BAR IntSer 112. Oxford 1981.

= M. H. CRAWFORD: Coinage and Money under the Roman Republic: Italy and the Mediterranean Economy. London 1985.

= PH. DAvis: Dacian imitations of Roman Republican denarii. Apulum 43 (2006) 321-356.

= S. FreY-KupPeR: Münzen. In: R. Zwahlen: Vicus Petinesca - Vorderberg. Die Holzbauphasen. Petinesca 2. Bern 2002, 129-144.

= D. GABLER: Die Eroberung Pannoniens im Spiegel der Sigillaten. ActaArchHung 23 (1971) 83-91.

$=$ D. GableR: Early Roman occupation in the Pannonian Danube Bend. In: Roman Frontier Studies 1995. Proceedings of the XVIth International Congress of Roman Frontier Studies, held at Kerkrade in the Netherlands in 1995. Eds: W. Groenman-van Waateringe, B. L. van Beek, W. J. H. Willems, S. L. Wynia. Oxbow monograph 91. Oxford 1997, 85-92.

= D. GABLER: Die Grabungen in der spätlatènezeitlich-römischen Siedlung von Budaörs: Augusteische Sigillaten in Budaörs. Italischer Import in der vorrömischen Zeit im pannonischen Raum. ActaArchHung 56 (2005) 133-175.

= D. GABLER: Das Donautal in augusteischer Zeit. Zur Frage der frühen Militärstationen. CarnuntumJb 2006 (2007) 81-101.

= D. GABLER: A budaörsi terra sigillaták (Terra Sigillata aus Budaörs). In: Római vicus Budaörsön. Régészeti tanulmányok. Hrsg.: K. Ottományi. Budapest 2012, 409-453.

= Ö. GoHL: A budapesti eraviszkusz éremlelet [Eravisker-Münzschatzfund in Budapest]. NK 1 (1902) $17-45$.

$=\mathrm{T}$. HABLE: Előzetes jelentés egy kelta (eraviszkusz) telep és római kori temető feltárásáról a vízivárosi katonai tábortól délre (Bp. I. ker. Corvin tér) - Preliminary report on the excavations at a Celtic (Eraviscus) settlement and a Roman period cemetery south of the Roman military fort in the Víziváros (Bp. District 1, Corvin Square). AqFüz 4 (1998) 25-38.

$=\mathrm{N}$. HANEL: Militär als Wirtschaftsfaktor in den Nordwestprovinzen in der frühen und mittleren Kaiserzeit. In: Das Militär als Kulturträger in römischer Zeit. Hrsg.: H. v. Hesberg. Köln 1999, 117-133

= P. HAUPT-M. Nick: Ein neuer Münzschatz mit Denaren der Eravisker aus der Gegend von Györ. NZ 104-105 (1997) 41-81.

= J. v. HeEsCH: Some considerations on the circulation of Augustan and Tiberian bronze coins in Gaul. In: Die Fundmünzen von Kalkriese und die frühkaiserzeitliche Münzprägung. Akten des wissenschaftlichen Symposions in Kalkriese, 15.-16. April 1996. Hrsg.: R. Wiegels. Osnabrücker Forschungen zu Altertum und Antike-Rezeption 3. Möhnesee 2000, 153-170.

= J. v. HeEsch: Les Romains et la monnaie gauloise: laisser-faire, laisser-aller? In: Die Kelten und Rom: neue numismatische Forschungen. Hrsg.: J. Metzler, D. Wigg-Wolf. Studien zu Fundmünzen der Antike 19. Mainz am Rhein 2005, 229-245.

$=$ J. HEINRICHS: Augusteische Truppen und niedergermanischer Geldumlauf. In: Das Militär als Kulturträger in römischer Zeit. Hrsg. : H. v. Hesberg. Schriften des Archäologischen Instituts der Universität Köln. Köln 2000, 147-187.

= J. JAHN: Zur Entwicklung römischer Soldzahlungen von Augustus bis Diocletian. In: Studien zu Fundmünzen der Antike. 2.: Aufsätze. Hrsg.: M. R.-Alföldi. Berlin 1984, 53-74.

= L. JUHÁSZ: Additional data on the gold hoard from Víziváros. In: Group and Individual Tragedies in Roman Europe. The Evidence of Hoards, Epigraphic and Literary Sources. Ed.: C. Gazdac. ClujNapoca 2020, 301-308.

= F. KEMMERs: Coins for a legion. An analysis of the coin finds from the Augustan legionary fortress and Flavian canabae legionis at Nijmegen. Studien zu Fundmünzen der Antike 21. Mainz am Rhein 2006.

= K. H. KÉRDỎ: Zur Frage der Lokalisierung des Auxiliarkastells von Víziváros. In: Roman Frontier Studies 1995. Proceedings of the XVIth International Congress of Roman Frontier Studies, held at Kerkrade in the Netherlands in 1995. Eds: W. Groenman-van Waateringe, B. L. van Beek, W. J. H. Willems, S. L. Wynia. Oxbow monograph 91. Oxford 1997, 399-404.

= K. H. KÉRDỎ: Das Alenlager und Vicus der Víziváros. In: Forschungen in Aquincum 1969-2002. Zu Ehren von Klára Póczy. Hrsg.: P. Zsidi. Aquincum nostrum II/2. Budapest 2003, 81-84. 
H. KÉRDÕ 2011

KING 1996

KOLNÍKOVÁ 2006

KovÁCS 2004

KovÁCS 2014

KovÁCs 2017

KRAAY 1962

KRAFT 1956

LOCKYEAR 1995

LOSCHEIDER 2005

LŐRINCZ 1990

MARÁz 2005

MrÁv 2004

MRÁv 2012

NASH 1976

NICK 2006

OTTOMÁNYI 2007

OTTOMÁNYI 2012

Peter 2001

Plachá-Fiala 1998

ROYMANS-AARTS 2009

SPEIDEL 1992

TORBÁGYI 1984

TORBÁGYI 1997/1998

TORBÁGYI 2003
= K. H. KÉRDŐ: Aquincum kezdetei és a településszerkezet változásai a Vízivárosban a római kor évszázadaiban. Régészeti adatok Aquincum-Víziváros történetéhez (Early ages of Aquincum and the changes in the settlement structure of the Viziváros during the centuries of Roman Ages). BudRég 44 (2011) 165-178.

= C. E. KING: Roman copies. In: Coin Finds and Coin Use in the Roman World. Eds: C. E. King, D. G. Wigg. Studien zu Fundmünzen der Antike 10. Berlin 1996, 237-263.

= E. KolníkovÁ: Münzen der Eravisker, Azaler und andere Nachahmungen der republikanischen Denare im nordkarpatischen Raum. In: In memoriam Ludovici Huszár. Hrsg.: K. Bíró Sey et al. Budapest 2006, 72-89.

= P. KovÁcs: The conquest of Pannonia and its organisation as a province. In: Early Geographers The Period of the Roman Conquest. Ed.: B. Fehér, P. Kovács. Fontes Pannoniae antiquae 1. Budapest 2005, 198-209.

= P. KovÁcs: A History of Pannonia during the Principate. Bonn 2014.

$=$ P. KovÁcs: Velleius Paterculus on Pannonia. In: Tiberius in Illyricum. Contributions to the History of the Danubian Provinces under Tiberius' Reign (14-37 AD). Ed.: P. Kovács. Hungarian polis studies 24. Budapest-Debrecen 2017, 103-119.

= C. M. KRAAY: Die Münzfunde von Vindonissa (bis Trajan). Veröffentlichungen der Gesellschaft pro Vindonissa 5. Basel 1962

= K. KRAFT: Das Enddatum des Legionslagers Haltern. BJ 155-156 (1955-1956) 96-111.

= K. LOCKYEAR: The supply of Roman Republican denarii to Romania. SCN 11 (1995) 85-102.

$=\mathrm{R}$. LOSCHEIDER: Militär und Romanisierung - Zum Münzwesen der Treverer nach dem Gallischen Krieg. In: Die Kelten und Rom: neue numismatische Forschungen. Hrsg. : J. Metzler, D. Wigg-Wolf. Studien zu Fundmünzen der Antike 19. Mainz am Rhein 2005, 109-125.

= B. LŌRINCZ: Legiok, segédcsapatok [Legionen, Hilfstruppen]. In: Pannonia régészeti kézikönyve. Hrsg.: A. Mócsy. Budapest 1990, 71-73.

= B. MARÁz: Budapest-Gellérthegy és környékének késő La Tène-kori településtörténete. 1.: Budapest-Tabán kelta-eraviszkusz telep topográfiai és településtörténeti meghatározásának kérdése (Siedlungsgeschichte von Budapest-Gellérthegy und Umgebung in der späten Latènezeit. 1.: Die Frage der topographischen und siedlungsgeschichtlichen Definition der keltisch-eraviskischen Siedlung von Budapest-Tabán). BudRég 39 (2005) 39-49.

= Zs. MrÁv: Loyalty and wealth: The native aristocracy of Roman Pannonia. In: Acts of the XIVth UISPP Congress, Liège 2-8 September 2001. Section 13.: Époque romaine / The Roman Age. BAR IntSer 1312. Oxford 2004, 1-11.

= Zs. MRÁv: Kora császárkori militaria a Budaörs-Kamaraerdei-dűlői vicus területéről (1st century militaria from the vicus at Budaörs-Kamaraerdei-dülö and the veterans settled in North-Eastern Pannonia). In: Római vicus Budaörsön. Régészeti tanulmányok. Hrsg.: K. Ottományi. Budapest 2012, 529-552.

= D. NASH: The growth of urban society in France. In: Oppida: The Beginnings of Urbanisation in Barbarian Europe. Papers presented to a conference at Oxford, October 1975. Eds: B. Cunliffe, T. Rowley. BAR SupplSer 11. Oxford 1976, 95-133.

= M. NICK: Gabe, Opfer, Zahlungsmittel. Strukturen keltischen Münzgebrauchs im westlichen Mitteleuropa. Freiburger Beiträge zur Archäologie und Geschichte des ersten Jahrtausends 12. Rahden 2006.

= K. OттомÁNYI: A pátyi római telep újabb kutatási eredményei (Die neuen Forschungsergebnisse der römischen Siedlung von Páty). StComit 30 (2007) 7-238.

= K. OtтomÁnYi: Római vicus Budaörsön (Der römische vicus von Budaörs). In: Római vicus Budaörsön. Régészeti tanulmányok. Hrsg.: K. Ottományi. Budapest 2012, 9-407.

= M. PETER: Untersuchungen zu den Fundmünzen aus Augst und Kaiseraugst. Studien zu Fundmünzen der Antike 7. Berlin 2001.

= V. PlachÁ-A. Fiala: Hromadný nález keltských minci hrade Devin (Hortfund keltischer Münzen auf der Burg Devín). SlovNum 15 (1998) 201-210.

$=$ N. ROYMANS - J. AARTS: Coin use in a dynamic frontier region. Late Iron Age coinages in the Lower Rhine area. Journal of Archaeology in the Low Countries 1-1 (May 2009) 5-26. www.jalc.nl and www.jalc.be

= M. A. SPEIDEL: Roman army pay scales. JRS 82 (1992) 87-106.

= M. ToRBÁGYi: Die Münzprägung der Eravisker. ActaArchHung 36 (1984) 161-196.

= M. TORBÁGYI: Elfelejtett római éremleletek III. (In Vergessenheit geratene römische Münzfunde III). NK 96-97 (1997-1998) 7-15.

= M. TORBÁGYI: Neue Beiträge zur eraviskischen Münzprägung. In: Pannonica provincialia et archaeologia. Studia sollemnia auctorum Hungarorum Eugenio Fitz octogenario dedicata. Ed.: Á. Szabó, E. Tóth. Libelli archaeologici SerN 1. Budapest 2003, 47-56. 
TORBÁGYI 2006

TORBÁGYI 2007

TORBÁGYI 2008

TORBÁGYI-VIDA 2013

VISY 2017

VONDROVEC 2007

WIGG 1996

WIGG 1997

WIGG 2002

WIGHTMAN 1974

WIGHTMAN 1977

WOLTERS 1988

WOLTERS 2001
= M. TORBÁGYI: Eraviszkusz „quinar” („Quinar“ der Eravisker). In: In memoriam Ludovici Huszár. Hrsg. : K. Bíró Sey et al. Budapest 2006, 90-95.

= M. TorbÁGYI: Páty-Malom-dűlő 1997-1999. évi ásatás érmeinek értékelése (Páty-Malom-dülő 1997-1999 - coin finds). StComit 30 (2007) 276-299.

= M. TORBÁGyI: Questions of coin use in the Late Celtic Transdanubia. Cercetări Numismatice 14 (2008) 203-212.

= M. ToRBÁGYI-I. VIDA: The coin hoard of Abasár. DissArch 3/1 (2013) 7-20.

= Zs. VISY: Zur Hypothese der mehrstufigen Einverleibung Pannoniens. In: Tiberius in Illyricum. Contributions to the History of the Danubian Provinces under Tiberius' Reign (14-37 AD). Ed. P. Kovács. Hungarian polis studies 24. Budapest-Debrecen 2017, 157-166.

= K. VondroveC: Gesamtdarstellung und Auswertung der antiken Fundmünzen im Museum Carnuntinum. In: Numismata Carnuntina. Forschungen und Materialien 1. Hrsg.: M. Alram, Fr. SchmidtDick. Denkschriften der philosophisch-historischen Klasse 353. Die Fundmünzen der römischen Zeit in Österreich 3/2. Veröffentlichungen zur Numismatik 44. Wien 2007, 57-340.

= D. G. WigG: The function of the last Celtic coinages in Northern Gaul. In: Coin Finds and Coin Use in the Roman World. Eds: C. E. King, D. G. Wigg. Studien zu Fundmünzen der Antike 10. Berlin 1996, 415-436.

= D. G. WIGG: Coin supply and the Roman army. In: Roman Frontier Studies 1995. Proceedings of the XVIth International Congress of Roman Frontier Studies, held at Kerkrade in the Netherlands in 1995. Eds: W. Groenman-van Waateringe, B. L. van Beek, W. J. H. Willems, S. L. Wynia. Oxbow monograph 91. Oxford 1997, 283-288.

= D. G. WIGG: Die Stimme der Gegenseite? Keltische Münzen und die augusteische Germanienpolitik. In: Kontinuität und Diskontinuität. Germania inferior am Beginn und am Ende der römischen Herrschaft. Hrsg.: T. Grünewald, S. Seibel. RGA - Ergänzungsband 35. Berlin-New York 2002, 218-241.

= E. M. Wightman: Le Gaule chevelue entre César et Auguste. In: Actes du IX Congrès International d'études sur les frontières romaines, Mamaia, 6-13 septembre 1972. Éd.: D. M. Pippidi. KölnBukarest-Wien 1974.

= E. M. Wightman: Soldier and civilian in early Roman Gaul. In: Limes. Akten des XI. Internationalen Limeskongresses (Székesfehérvár, 30.8.-6.9.1976). Hrsg.: J. Fitz. Budapest 1977, 75-86.

$=$ R.WOLTERS: Keltische Münzen in römischen Militärstationen und die Besoldung römischer Hilfstruppen in spätrepublikanischer und frühaugusteischer Zeit. Tyche 3 (1988) 261-272.

$=$ R. Wolters: Bronze, silver or gold? Coin finds and the pay of the Roman army. Zephyrus 53-54 (2000-2001) 579-588.

Open Access. This is an open-access article distributed under the terms of the Creative Commons Attribution 4.0 International License (https:// creativecommons.org/licenses/by/4.0), which permits unrestricted use, distribution, and reproduction in any medium, provided the original author and source are credited, a link to the CC License is provided, and changes - if any - are indicated. (SID_1) 
